\title{
МОДЕЛЬ ДИНАМИКИ БИОЛОГИЧЕСКОЙ НЕЙРОННОЙ СЕТИ С ГИСТЕРЕЗИСНЫМИ СВЯЗЯМИ
}

\author{
А. М. Соловьёв ${ }^{*}$ Е. Г. Кабулова ${ }^{\star *}$, М. Е. Семёнов \\ ${ }^{*}$ Воронежский государственный университет \\ ${ }^{*}$ Старооскольский технологический институт им. А.А. Угарова (филиал) НИТУ «МИСиС»
}

\author{
Поступила в редакцию 12.03.2018 г.
}

\begin{abstract}
Аннотация. Проведено исследование динамики биологической нейронной сети, построенной на основе модифицированной математической модели С.А. Кащенко - В.В. Майорова. Выполнено моделирование запуска эндогенных процессов, образования спайка в синапсе нейрона, а также синхронизации нейронных ансамблей под воздействием метаботропного рецептивного кластера с учетом гистерезисных связей и типа воздействия (химического или электрического). Для описания гистерезисных зависимостей, возникающих в процессе работы нейросети, используется феноменологическая модель Боука-Вена.

Ключевые слова: биологический нейрон, нейронная сеть, синхронизация нейронов, гистерезис, модель Боука-Вена.

Annotation. In this work we investigated the dynamic of biological neural networks based on modified mathematical model of Kashchenko - Maiorov. We performed a simulation launch of endogenous processes, the formation of membrane depolarization of the neuron in the synopsis, and synchronization of neuronal ensembles under the influence of metabotropic receptive cluster, subject to hysteresis connexions and the type of impact (chemical or electrical). To describe the hysteresis dependencies that arise in the process of the neural network working, we used a phenomenological model of Bouc-Wen.

Keywords: biological neuron, neural network, synchronization of neurons, hysteresis, BoucWen model.
\end{abstract}

\section{ВВЕДЕНИЕ}

Интерес к исследованию нейронных сетей связан в первую очередь с тем, что способ обработки информации человеческим мозгом кардинально отличается от методов, применяемых в современных компьютерах. Мозг является чрезвычайно сложной, нелинейной, параллельной системой обработки информации. Он обладает способностью организовывать свои структурные компоненты (нейроны) таким образом, чтобы они могли выполнять специализированные задачи во много раз быстрее, чем самые быстродействующие современные компьютеры.

В настоящее время в теории нейронных сетей активно развивается направление по изучению осцилляторных аспектов функ2018 ционирования головного мозга. Существует ряд математических моделей [1-6], позволяющих описать взаимодействие нейронов в коре головного мозга. Данные модели имеют разную степень своей биологической обоснованности. Одной из наиболее обоснованных и приближенных к биологическим данным математических моделей, является модель Ходжкина-Хаксли $[7,8]$. Однако, главным недостатком этой модели является сложность ее использования для постановки численных экспериментов, вследствие чего она практически не используется для моделирования нейронных сетей.

В настоящей работе проводится исследование динамики нейронов биологической нейронной сети, построенных на основе модифицированной математической модели С. А. Кащенко - В. В. Майорова [1], обладающей высокой биологической обоснованностью. Следует отметить, что построенная в 


\section{А. М. Соловьёв, Е. Г. Кабулова, М. Е. Семёнов}

работе математическая модель отражает особенности связей между отдельными биологическими нейронами, показанные в работе А. Н. Радченко [9]. Согласно исследованиям Радченко, каждый биологический нейрон окружен клеточными образованиями (кластерами), которые при определенных условиях способны запустить эндогенные процессы в нейроне, за которыми может последовать спайк. При этом, запуск эндогенных процессов имеет гистерезисную природу.

В настоящей работе проводится исследование динамики биологических нейронов в зависимости от структуры нейронных сетей, а также связей между ними.

\section{МОДЕЛЬ НЕЙРОНА КАЩЕНКО-МАЙОРОВА}

Феноменологическая модель Кащенко Майорова основана на течении через мембрану нейронов калиевых и натриевых токов. Данная модель применялась в работе [1] для моделирования кольцевых нейронных структур, в которых возбуждение нейронов-предшественников волнообразно передавалось на нейроны-последователи. Важной особенностью модели Кащенко-Майорова является возможность описания с помощью нее эффекта запаздывания калиевых токов от натриевых, наблюдаемого в биологических нейронах.

Функция активации нейрона в данной модели описывается дифференциальным уравнением с запаздыванием следующего вида:

$$
\dot{u}=\lambda(-1+f K(u(t-1))-f N a(u)) u,
$$

с соответствующим начальным условием:

$$
\left.u(t)\right|_{-1 \leq t \leq 0}=\varphi(t),
$$

где $\lambda=\frac{h(b-a)}{c}, a, b, c-$ коэффициенты, отражающие биологические свойства мембраны нейрона, $h$ - время запаздывания калиевых токов от натриевых, $f K(u)$ и $f N a(u)$ характеризуют калиевый и натриевый токи. На эти функции накладываются следующие ограничения:

$$
\begin{aligned}
& f K(u)>0, f N a(u)>0, \\
& -1-f N a(0)+f K(0)>0, \\
& f K(u)<C u^{-1-\varepsilon}, \\
& f N a(u)<\mathrm{Cu}^{-1-\varepsilon},
\end{aligned}
$$

где $\varepsilon>0$, что следует из биологической обусловленности модели.

Нейрон подвергается воздействию как электрической, так и химической природы. В случае возбуждения нейрона от электрического воздействия, функция активации (1) будет иметь вид

$$
\dot{u}=\lambda(-1+f K(u(t-1))-f N a(u)) u+g(t),
$$

где $g(t)$ - интенсивность электрического воздействия.

В случае возбуждения нейрона от химического воздействия, функция активации описывается следующим уравнением:

$$
\dot{u}=\lambda(-1+f K(u(t-1))-f N a(u)+v(t)) u .
$$

где $v(t)$ - интенсивность химического воздействия.

Как было показано в работе [1], химическое воздействие эффективно при моделировании связи и синхронизации ансамблей в нейронной сети, электрическое же воздействие эффективно навязывает период генерации спайков.

\section{МОДЕЛЬ ПАМЯТИ НЕЙРОНОВ РАДЧЕНКО}

Как известно, способность биологических нейросетей воспринимать и хранить информацию непосредственно связана с обучением. В случае классических искусственных нейронных сетей, данная задача решается с помощью подбора коэффициентов из матрицы связей между нейронами (например, методом обратного распространения ошибок). В работе [9] была предложена модификация классической модели нейросети, в которой характер связи между нейронами имеет гистерезисную природу, что согласуется с биологическими данными. Также, в данной работе были проведены биологические эксперименты, в ходе которых обнаружены специальные образования вокруг синапсов нейронов - метаботроп- 
ный рецептивный кластер (МРК). Данное образование может воздействовать на нейрон, запуская внутренние химические процессы, провоцируя появление спайка. Следует также отметить, что гистерезисная природа запуска внутренних процессов зависит от характера воздействия на нейрон (электрического или химического).

\section{ХИМИЧЕСКОЕ ВОЗДЕЙСТВИЕ НА МРК}

В случае возбуждения нейрона от химического воздействия, запуск эндогенных процессов посредством МРК будет происходить как показано на рис. 1.

Гистерезисные кривые, приведенные на рис. 1, можно описать с помощью следующего уравнения:

$$
u=B y \sqrt{\frac{1-k}{y-k}}-A y^{2},
$$

где $A=\frac{\lambda_{0} \rho}{\varepsilon}, B=\lambda_{0} \sqrt{\frac{2 Y}{\varepsilon}}$, и полученные экспериментальным путем значения констант:

$$
\begin{aligned}
& \lambda_{0}=1.4 * 10^{-9}, \varepsilon=8.85 * 10^{-11}, \\
& Y=0.67 * 10^{6} .
\end{aligned}
$$

Форма кривой, изображенной на рис. 1 зависит от коэффициента $k(0 \leq k \leq 1)$. С биологической точки зрения данный параметр определяет подвижность зарядов в МРК.

При увеличении воздействия $u_{m}$ на МРК происходит переход с верхней части кривой на нижнюю (рис. 1) вследствие гиперполяризационного конформационного перехода (ГКП). Данный эффект с биологической точки зрения объясняется тем, что частицы рецептивного кластера уплотняются, накапливая энергию. При дальнейшем увеличении $u_{m}$, коэффициент $k$ стремится к единице, вследствие чего продолжается уплотнение МРК, при этом кривая меняется от $A_{0}$ до $A_{3}$.

Далее, в случае уменьшения воздействие $u_{m}$ до величины, достаточной для обратного перехода с нижней части кривой на верхнюю, произойдет химический конформационный переход (ХКП). При этом на нейрон будет воздействовать накопленная энергия в МРК после ГКП.

Из описанного механизма работы МРК можно сделать вывод, что периодическое химическое воздействие с растущей амплитудой будет наиболее эффективным, подобно воздействию со стороны нейронного ансамбля, притягивающего к себе нейроны и тем самым увеличивающего силу притяжения.

\section{ЭЛЕКТРИЧЕСКОЕ ВОЗДЕЙСТВИЕ}

В случае возбуждения нейрона с помощью электрического воздействия, уравнение (5) перепишется:

$$
u=B y \sqrt{\frac{1-k}{y-k}},
$$

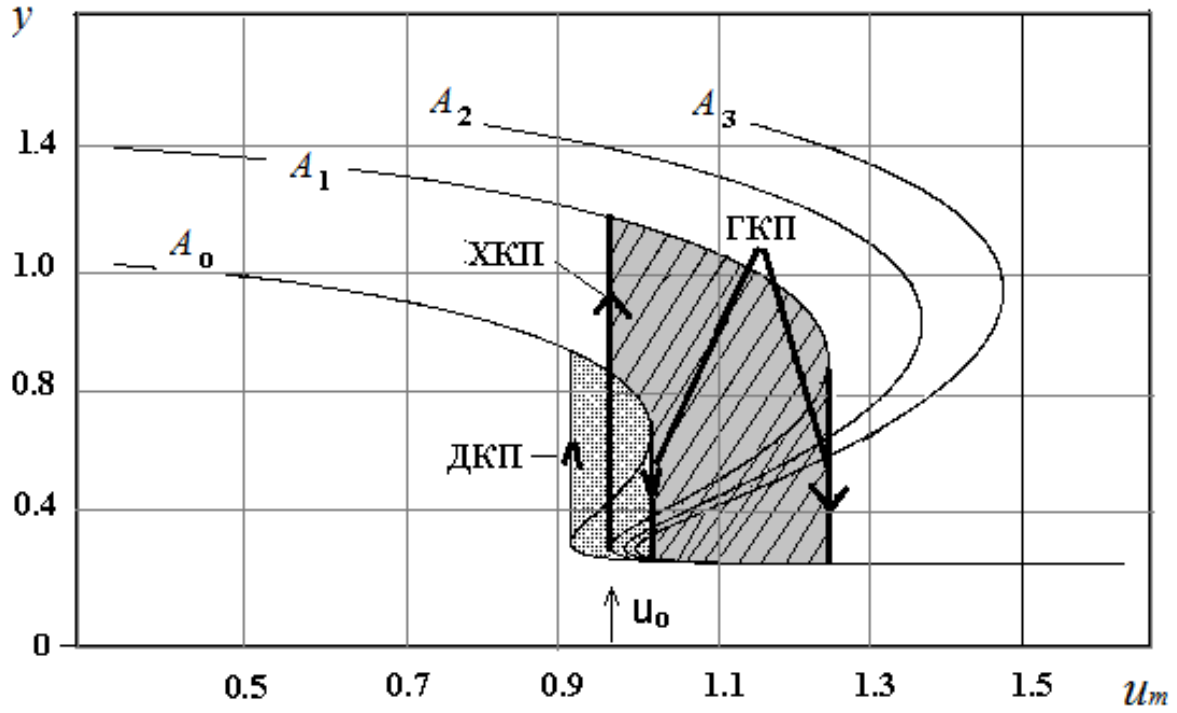

Рис. 1. Изменение формы гистерезисных зависимостей, описывающих динамику МРК в случае химического воздействия 


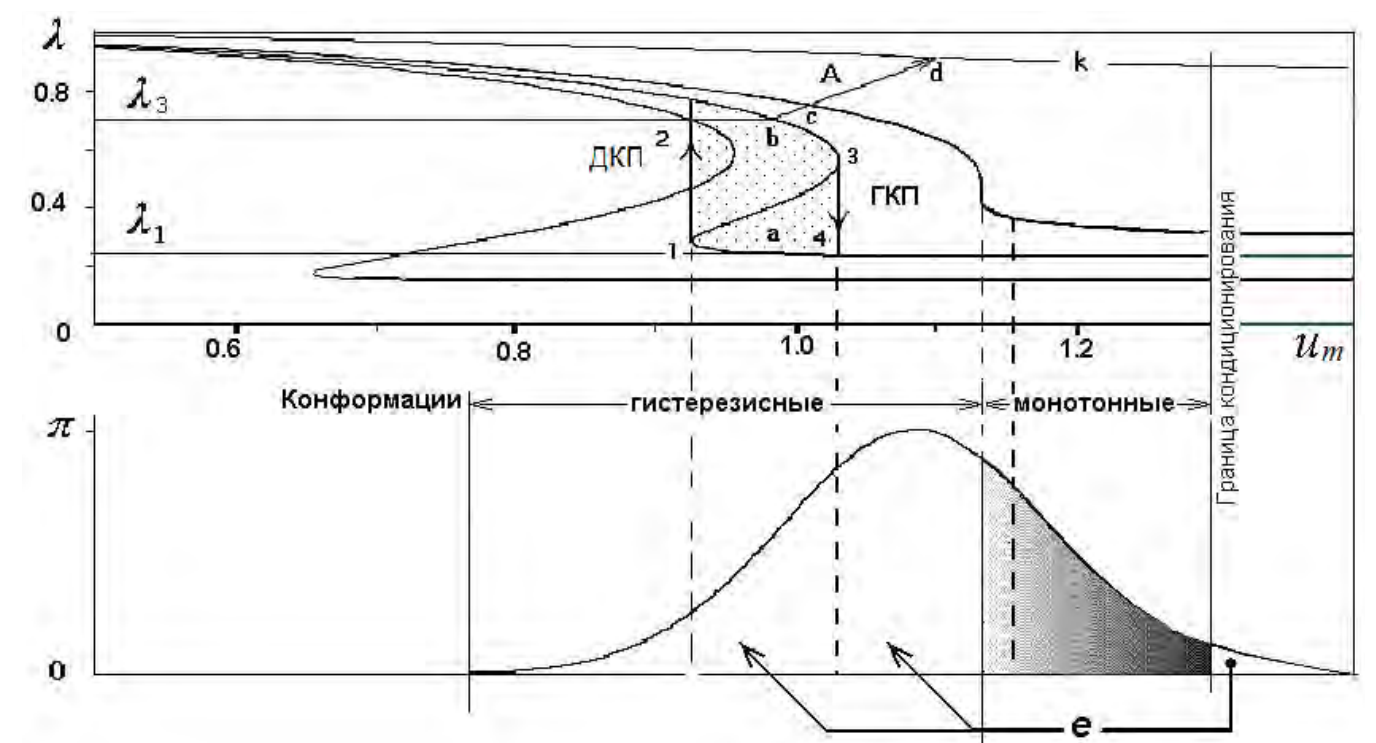

Рис. 2. Изменение формы гистерезисных кривых, описывающих динамику МРК в случае электрического воздействия

Изменение формы гистерезисных гистерезисных кривых в зависимости от коэффициента $k$ приведено на рис. 2.

Как видно из рис. 2 , рост величины воздействия $u_{m}$ МРК ведет к потере ее гистерезисной природы, в процессе чего утрачивается способность к запуску внутренних процессов в нейроне (изменение кривой $a \rightarrow b \rightarrow c \rightarrow d$ ). Таким образом, воздействие МРК на нейрон значительно ослабляется под воздействием сильной электрической стимуляции.

\section{ОПИСАНИЕ МОДЕЛИ}

Рассмотрим искусственную нейронную сеть, отдельные элементы которой описываются следующим дифференциальным уравнением:

$\dot{u}_{i}=\lambda\left(-1+f K\left(u_{i}(t-1)\right)-f N a\left(u_{i}\right)+Y_{i}\right)+I_{i}$,

где $Y_{i}$ - воздействие со стороны нейронной сети, оказываемое на $i$ нейрон, $I_{i}$ - внешнее воздействие на $i$ нейрон. Величина $Y_{i}$ задает химическую связь между элементами нейронной сети, которая описывается с помощью модели Радченко, а $I_{i}$ определяет внешнее воздействие электрической природы.

Пусть

$$
Y_{i}=\left\{\begin{array}{l}
y_{i}, \text { если } \dot{y}_{i}>\gamma \\
0, \text { востальныхслучаях }
\end{array},\right.
$$

где $\gamma$ - пороговая скорость роста $y_{i}$. Данное ограничение введено для определения этапа
ХКП, когда $y_{i}$ быстро растет. Связь между нейронами сети количественно можно оценить с помощью следующего равенста:

$$
x_{i}(t)=\sum_{j=1}^{N} D_{i j} \frac{\int_{t-T}^{t}\left|u_{i}-u_{j}\right| d s}{\int_{t-T}^{t} u_{i} d s},
$$

где $D_{i j}$ - коэффициент силы связи между $i$ и $j$ нейронами, $N+1$ - общее количество нейронов. Параметр $T$ необходимо подбирается таким образом, чтобы на соответствующем промежутке укладывался ровно один спайк. По определению, значение величины $x_{i}(t)$ должно возрастать вместе с интегральной мерой рассинхронизации нейронов.

Отметим, что связь между величинами $y_{i}$ и $X_{i}$ носит гистерезисный характер. В качестве математической модели, описывающей данную гистерезисную зависимость, используем феноменологическую модель Боука-Вена [10-12], отличающуюся простотой математического описания и численной реализации. Согласно модели Боука-Вена, зависимость величин $y_{i}$ и $x_{i}$ будет определяться следующим дифференциальным уравнением:

$$
\dot{y}_{i}=B \dot{x}-\beta\left|\dot{x}_{i}\right|\left|y_{i}\right|^{n-1} y_{i}-\alpha \dot{x}_{i}\left|y_{i}\right|^{n},
$$

где коэффициенты $B, n, \beta$ и $\alpha$ определяют форму гистерезисной зависимости.

Внешнее электрическое воздействие запишем как 
Модель динамики биологической нейронной сети с гистерезисными связями

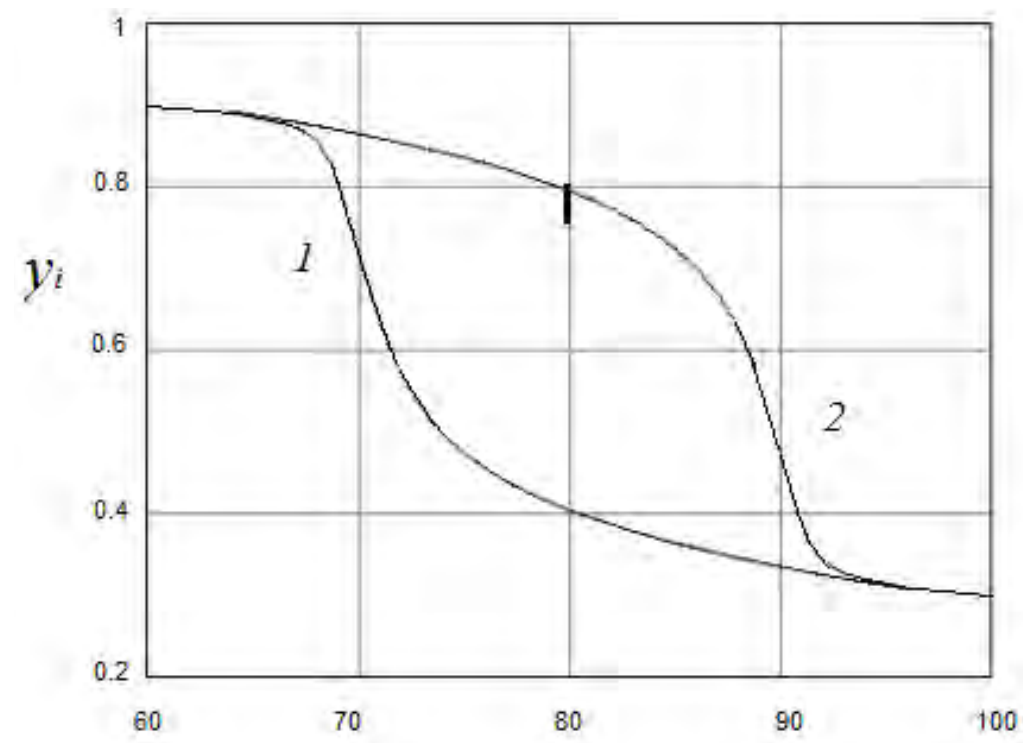

Рис. 3. Динамика у при периодическом входе

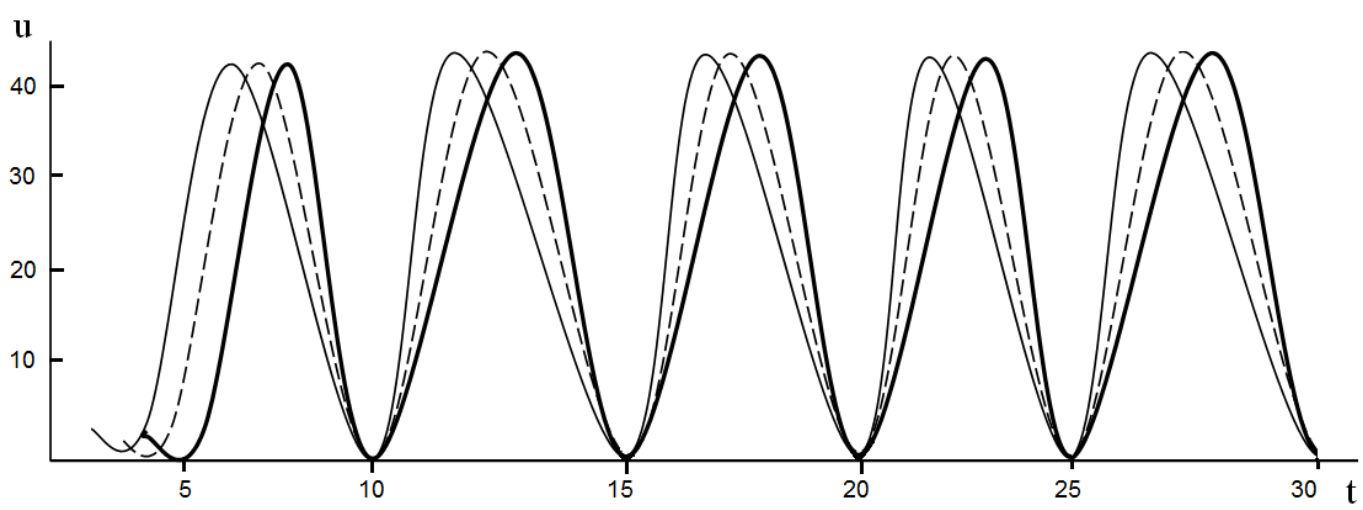

Pис. 4. Динамика трех нейронов с $D_{i j}=0.01$

$$
\begin{aligned}
I_{i}= & \left\{\begin{array}{l}
y_{i}, \text { если } \dot{y}_{i}>\gamma \\
0, \text { востальныхслучаях }
\end{array},\right. \\
& x_{i}=A e^{-\alpha t} \sin \left(t-g_{i}\right),
\end{aligned}
$$

где $g_{i}$ - внешнее воздействие на $i$ нейрон, $A$ положительная константа.

Изменение $y_{i}$ при условии, что $x_{i}=15 \sin (t)+80$ и фиксированных коэффициентах $B, n, \beta, \alpha$ показана на рис. 3 .

Как было показано в работе [9], МРК способен вызвать спайк нейрона, в случае, когда его динамика описывается кривой 1 на рис .2. То есть, пока воздействие на нейрон остается постоянным или возрастает, нейрон будет реагировать на него без посредников. Однако, если это воздействие начнет уменьшаться, спайк нейрона будет обусловлен МРК.

\section{ИССЛЕДОВАНИЕ НЕЙРОННОЙ СЕТИ СО «СЛАБОЙ» СВЯЗЬЮ}

Проведем исследование искусственной нейронной сети с входными воздействиями (11) на каждом ее элемент. При этом будем считать, что внешние воздействия нормированы условием

$$
0 \leq g_{i} \leq 1
$$

и равны, соответственно, 0.1, 0.5, 0.8 .

Рассмотрим случай, когда величина $D_{i j}$ из равенства (9) достаточно мала. При этом влияние на динамику сети связи между нейронами практически полностью нивелируется. Результаты моделирования в этом случае показаны на рис. 4.

Вследствие наличия гистерезиса во входных воздействиях, фазовые портреты динамики нейронов сети будут иметь вид, приведенный на рис. 5. 
А. М. Соловьёв, Е. Г. Кабулова, М. Е. Семёнов
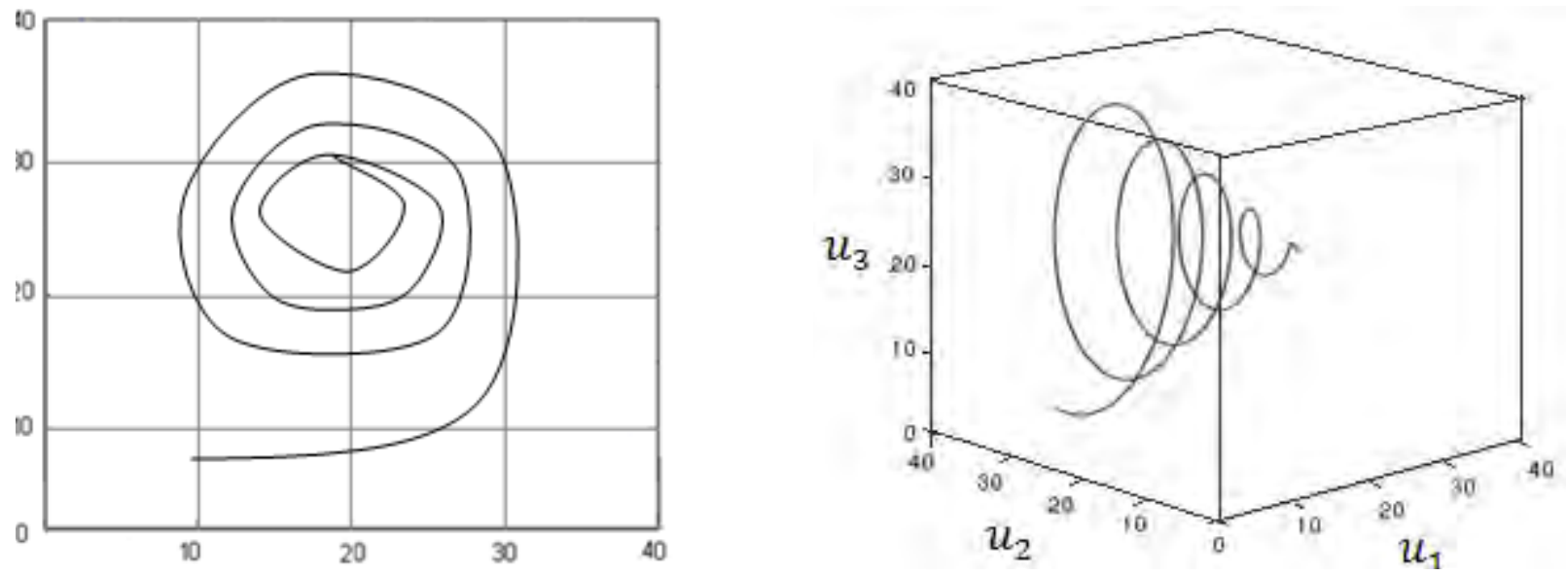

Puс. 5. Фазовые портреты системы (6) при $D_{i j}=0.01$

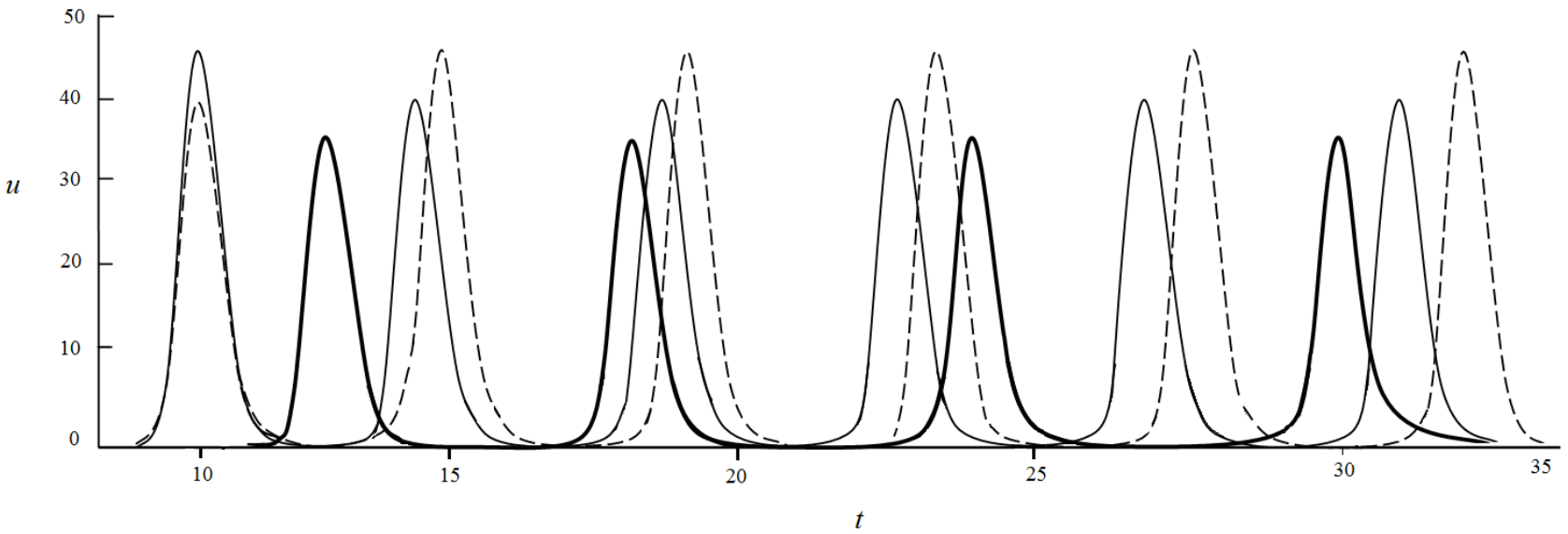

Рис. 6. Динамика трех нейронов при выполненном условии (14)
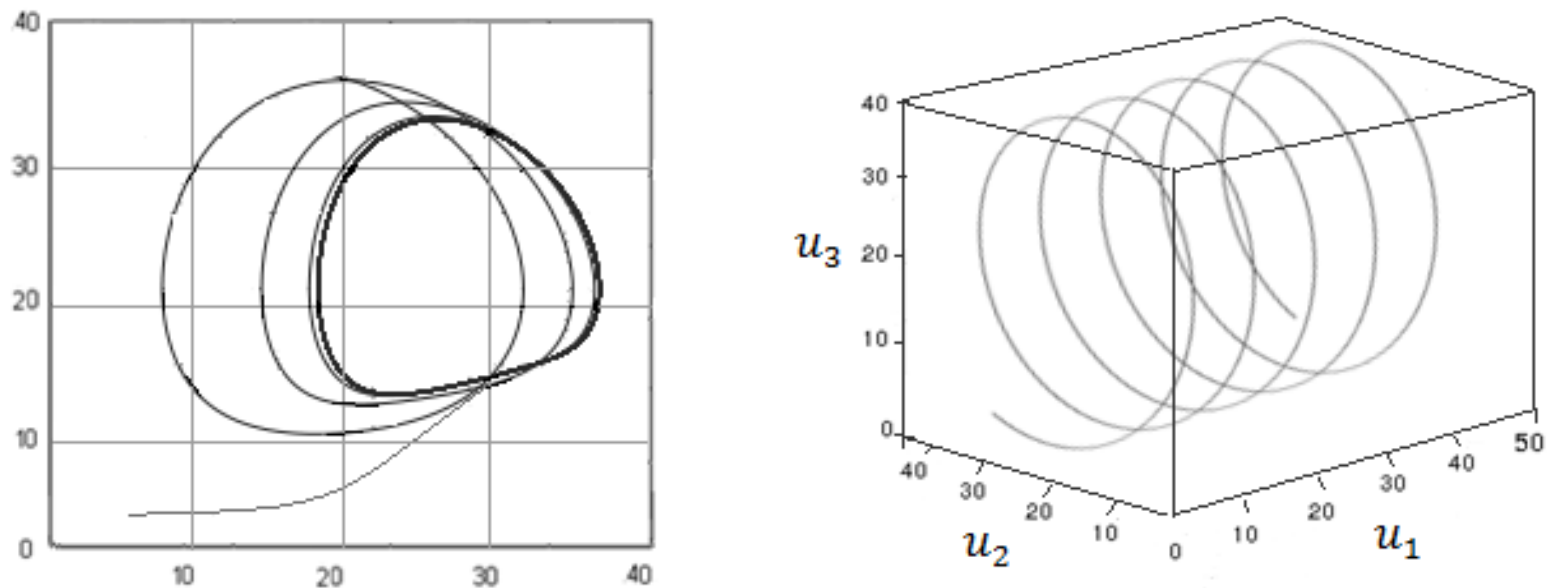

Рис. 7. Фазовые портреты системы (6) при выполненном условии (14)

Как видно из рисунков, эффект синхронизации нейронов отсутствует на достаточно продолжительном промежутке времени.

В случае, когда во входном воздействии отсутствуют гистерезисные зависимости и выполняется условие, приведенное в работе [1],

$$
I_{i}=g_{i},
$$

динамика нейронной сети примет вид, изображенный на рис. 6, 7.

Сравним графики на рис. 4,5 и рис. 6,7 соответственно. Как видно из рисунков, гистерезис вносит упорядочивающий эффект в нейронную сеть, что может быть полезным при решении задач сегментации, классификации и распознавания образов. 


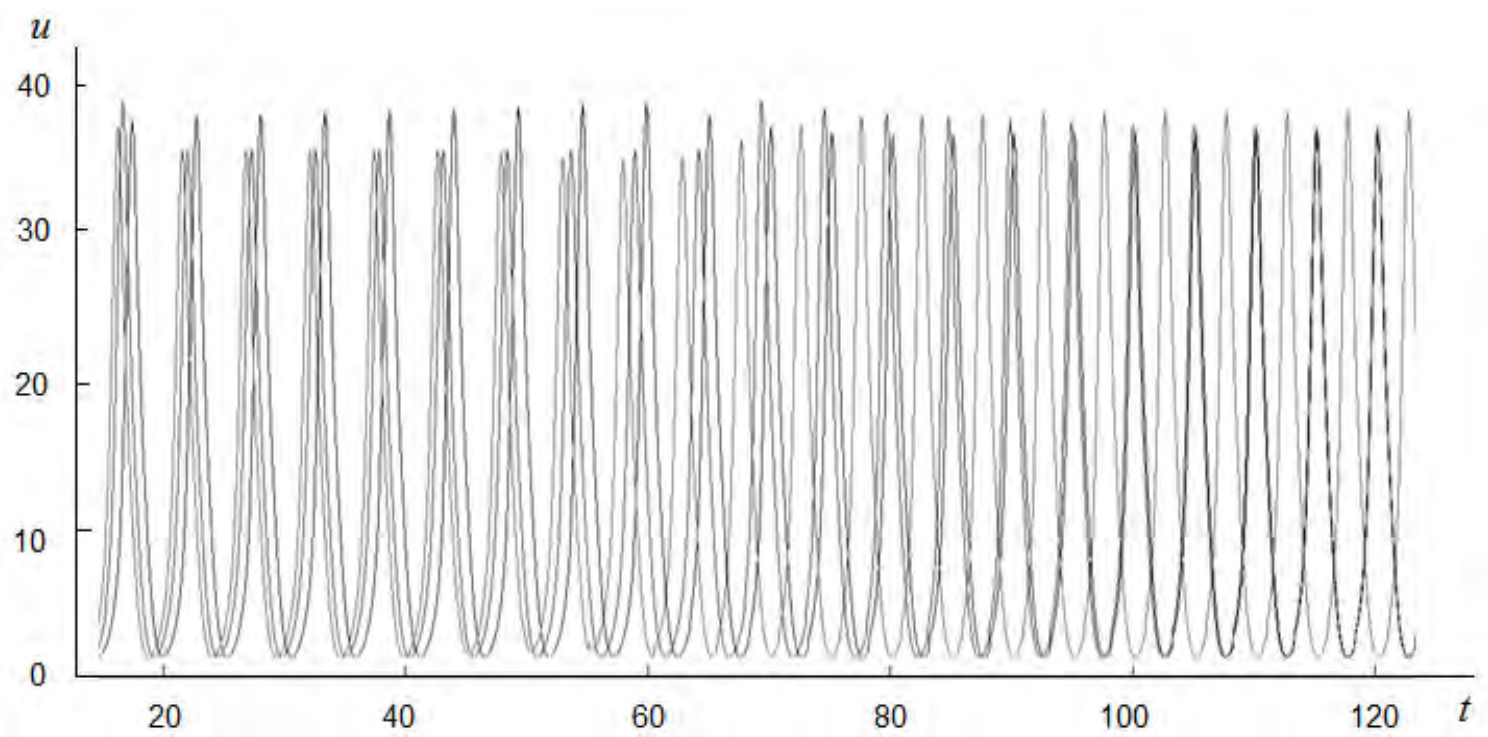

Рис. 8. Динамика нейронной сети с входными воздействиями 0, 0.5, 1

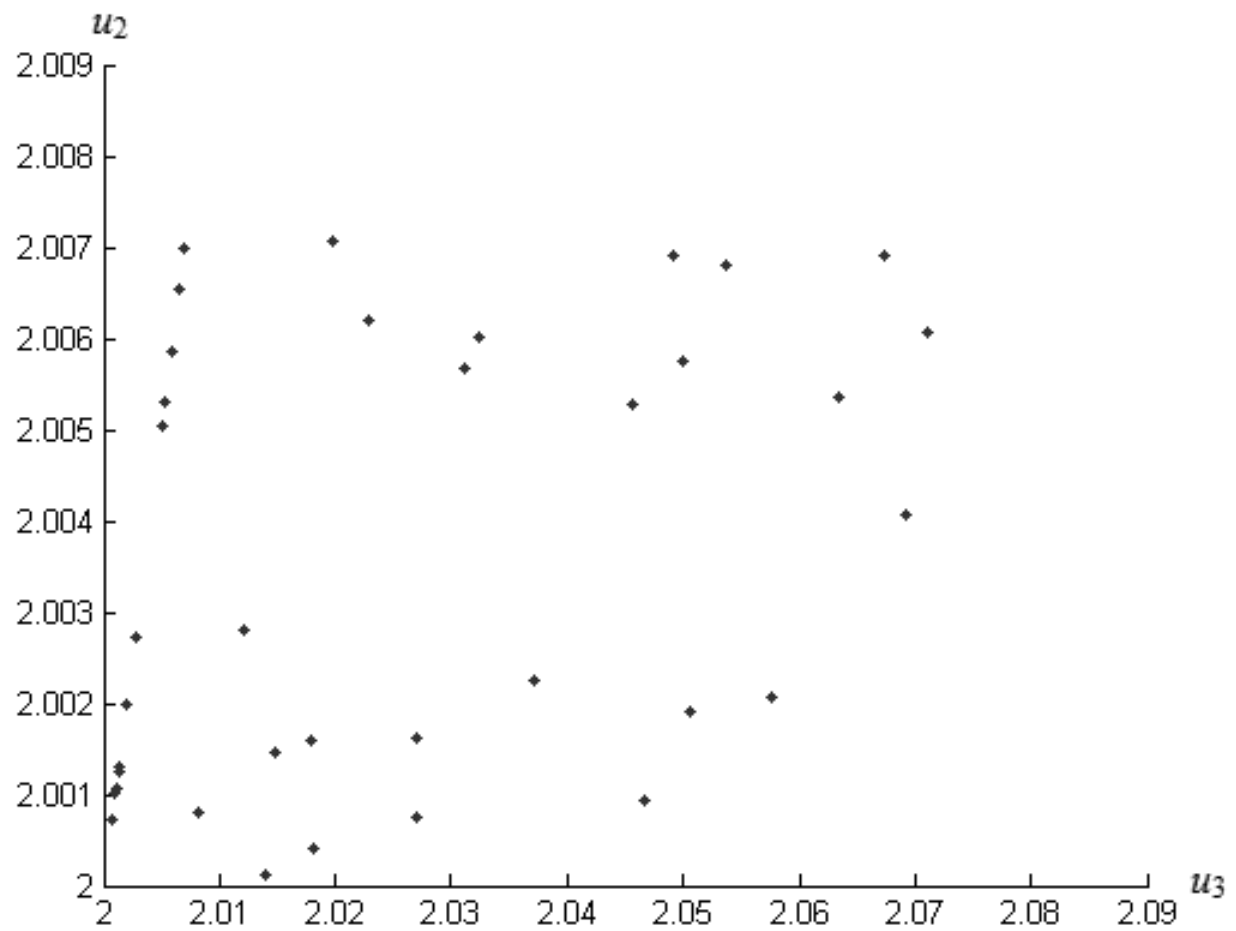

Рис. 9. Сечение Пуанкаре фазовых траекторий системы из трех нейронов

\section{ИССЛЕДОВАНИЕ НЕЙРОННОЙ СЕТИ \\ С «СИЛЬНОЙ» ПОСТОЯННОЙ ГИСТЕРЕЗИСНОЙ СВЯЗЬЮ}

Проведем исследование искусственной нейронной сети, состоящей из трех нейронов, которая описывается системой дифференциальных уравнений (7). Будем считать, что гистерезисная связь между нейронами активна и неизменна, то есть

$$
k_{i}=\text { const }, \quad 0<k_{i}<1 \text {. }
$$

Результаты численного моделирования данной нейронной сети приведены на рис. 8, 9.

Как видно из рис. 8, при стабильности гистерезисной связи, динамика одного из нейронов приводит к синхронизации остальных элементов сети, начиная с 100 секунды. Данный эффект является биологически обоснованным, что следует из результатов работы [13]. Наличие синхронизации объясняется тем, что нейрон с наибольшим внешним воздействием становится центральным эле- 
ментом в сети. В этом случае вся сеть настраивается на восприятие наиболее сильного внешнего воздействия, а остальные воздействия игнорируются.

Согласно работе [9] возврат гистерезисной природы МРК, а значит и способность запоминания информации, происходит во время сна. Таким образом, моделирование нейронной сети (7) при выполнении условия (15) может описывать работу мозга в состоянии сна. Согласно [14], основная задача мозга во сне - консолидация полученной информации во время бодрствования. При этом запомнится информация, соответствующая наиболее сильному раздражителю. Таким образом, можно предположить, что у системы (7) при постоянной гистерезисной связи существует аттрактор.

Проведем исследование динамики нейронной сети (7) при внешних воздействиях вида

$$
\begin{aligned}
& g_{1}=1, \\
& 0.4 \leq g_{2} \leq 1, \\
& 0.4 \leq g_{3} \leq 1 .
\end{aligned}
$$

Построим сечение Пуанкаре фазовых траекторий с плоскостью $u_{1}=0.4$ (рис. 9).

Как видно из рисунка $u_{2}(t) \approx u_{3}(t)$, что можно объяснить появлением в нейронной сети центрального элемента. Подробное исследование динамики нейронных сетей с центральным элементом было проведено в работе [15].

\section{ЗАКЛЮЧЕНИЕ}

Рассмотренная в настоящей работе математическая модель нейронной сети показывает, что даже при небольшом количестве нейронов ее динамика согласуется с биологическими данными [14]. Преимуществом предложенной в работе модели является ее «упорядоченная» реакция на внешние воздействия. Данная особенность привносится за счет использования модели [9], что позволяет моделировать нейронную сеть с множеством элементов [16]. При этом достигается синхронизация нейронов в ансамбли $[17,18]$. Отметим, что описанная в работе нейронная сеть может быть использована для решения задач сегментации и классификации образов.

Работа поддержана РФФИ: гранты № 1701-00251 u 16-08-00312

\section{СПИСОК ЛИТЕРАТУРЫ}

1. Кащенко С. А. Модели волновой памяти / С. А. Кащенко, В. В. Майоров. - М. : УРСС, 2009. - 288 c.

2. FitzHygh R. Biophys. J. - 1961. - 1445.

3. Nagumo J., Arimoto S., Yoshizawa S. Proc. IRE. - 1962. - 502061.

4. Hodgkin A. L., Huxley A. F. J. Physiol. (London). - 1952. - 117500.

5. Hansel D., Mato G., Meunier C. Neural Comp. - 1995. - 7307.

6. Guoshen Yu and Jean-Jacques Slotine. Visual Grouping by Neural Oscillator Networks // Neural Networks, IEEE Transactions on. - 2009. 20(12). - P. 1871-1884.

7. Hodgkin A. L., Huxley A. F. J. Physiol. (London). - 1952. - 117500.

8. Hansel D., Mato G., Meunier C. Neural Comp. - 1995. - 7307.

9. Радченко А. Н. Ионотропные и метаботропные реакции нейрона как инструменты нейронной памяти // Нейроинформатика. - 2006. - Т. 1, № 2. - С. 197-227.

10. Ikhouane F., Manosa V., Rodellar J. Dynamic properties of the hysteretic Bouc-Wen model / F. Ikhouane, V. Manosa, J. Rodellar // Systems \& Control Letters. - 2007. - V. 56. P. 197-205.

11. Ikhouane F., Rodellar J. On the Hysteretic Bouc-Wen Model / F. Ikhouane, J. Rodellar // Nonlinear Dynamics - 2005. - V. 42. - P. 63-78.

12. Solovyov A. M., Semenov M. E., Meleshenko P. A., Barsukov A. I. Bouc-Wen model of hysteretic damping // Procedia Engineering. 2017. - 201. - P. 549-555.

13. Казанович Я. Б., Борисюк Р. М. Синхронизация в нейронной сети фазовых осцилляторов с центральным элементом // Матем. моделирование. - 1994. - 6:8. - 45-60.

14. Sara J. Aton, Julie Seibt. Neuron 61, 454466, February 12, 2009 Elsevier Inc. 
15. Крюков В. И. Модель внимания и памяти, основанная на принципе доминанты и компараторной функции гиппокампа // Журнал высшей нервной деятельности. - 2004. № 1 .

16. Грачиков Д. В., Семенов М. Е., Канищева О.И. Синхронизация нейронных ансамблей под действием МРК // XIII Международная конференция «Информатика: проблемы, методология, технологии», 2013.

Соловьёв А. М. - аспирант кафедры цифровых технологий, факультет компьютерных наук, Воронежский государственный университет.

E-mail: darkzite@yandex.ru

Кабулова Е. Г. - доцент кафедры высшей математики, Старооскольский технологический институт им. А.А. Угарова (филиал) НИТУ «МИСиС».

E-mail: evgenia791@mail.ru

Семёнов М. Е. - д-р физ.-мат. наук, профессор кафедры цифровых технологий, факультет компьютерных наук, Воронежский государственный университет.

E-mail:mkl150@mail.ru
17. Абарбанель Г. Д., Рабинович М. И., Селверстон А., Баженов М. В., Хуэрта.Р, Сущик М. М., Рубчинский Л. Л. Синхронизация в нейронных ансамблях // УФН. - 1996. - 166 363-390.

18. Лебедев Г.Н., Семенов М. Е., Грачиков Д. В., Канищева О. И. Гистерезисная модель синхронизации биологических нейронов // Информационные технологии. - 2014. - № 6. C. 11-16.

Solovyov A. M. - graduate student of Digital Technologies Department, Voronezh State University.

E-mail: darkzite@yandex.ru

Kabulova E. G. - associate professor, department of mathematics, Stary Oskol Technological Institute named after A.A. Ugarov (branch) National University of Science and Technology «MISIS». E-mail: evgenia791@mail.ru

Semenov M. E. - doctor of physical and mathematical Sciences, professor of Digital Technologies Department, Voronezh State University. E-mail:mkl150@mail.ru 